\title{
Padovan numbers which are palindromic concatenations of two distinct repdigits
}

\author{
Taboka P. Chalebgwa ${ }^{1}$ (D) Mahadi Ddamulira ${ }^{2,3,4}$ (ID) \\ Received: 5 September 2020 / Accepted: 9 April 2021 / Published online: 24 April 2021 \\ (c) The Author(s) 2021
}

\section{Abstract}

In this paper we determine all Padovan numbers that are palindromic concatenations of two distinct repdigits.

Keywords Padovan numbers · Repdigits · Linear forms in logarithms · Baker-Davenport reduction method

Mathematics Subject Classification 11B39 $\cdot$ 11D61 $\cdot$ 11J86

\section{Introduction}

Let $\left(P_{n}\right)_{n \geq 0}$ be the sequence of Padovan numbers, given by $P_{n+3}=P_{n+1}+P_{n}$, for $n \geq 0$, where $P_{0}=0$ and $P_{1}=P_{2}=1$. The first few terms of this sequence are

$$
\left(P_{n}\right)_{n \geq 3}=\{1,2,2,3,4,5,7,9,12,16,21,28,37,49,65,86,114,151,200, \ldots\} .
$$

A repdigit (in base 10) is a positive integer $N$ that has only one distinct digit. That is, the decimal expansion of $N$ takes the form

$$
N=\underbrace{\overline{d \cdots d}}_{\ell \text { times }}=d\left(\frac{10^{\ell}-1}{9}\right),
$$

\section{Mahadi Ddamulira}

mddamulira@tugraz.at; mddamulira@mpi-sws.org; mahadi.ddamulira@mak.ac.ug

Taboka P. Chalebgwa

chalebgt@mcmaster.ca

1 Department of Mathematics and Statistics, McMaster University, Hamilton, ON L8S 4K1, Canada

2 Institute of Analysis and Number Theory, Graz University of Technology, Kopernikusgasse 24/II, 8010 Graz, Austria

3 Max Planck Institute for Software Systems, Saarland Informatics Campus, Campus E1 5, 66123 Saarbrücken, Germany

4 Department of Mathematics, School of Physical Sciences, College of Natural Sciences, Makerere University, PO Box 7062, Kampala, Uganda 
for some positive integers $d$ and $\ell$ with $0 \leq d \leq 9$ and $\ell \geq 1$. This paper is a contribution to the rather well studied topic of Diophantine properties of certain linear recurrence sequences. More specifically, our paper is a variation on the theme focusing on representations of terms of a recurrent sequence as concatenations of members of another (possibly the same) sequence. For a general study of the results underpinning this topic, we direct the reader to the paper [2] by Luca and Banks, wherein (as a consequence of their level of generality) some ineffective (but finiteness) results were obtained on the number of terms of certain binary recurrent sequences whose digital representation consists of members of the same sequence.

In Ref. [1], the authors considered Fibonnaci numbers which are concatenations of two repdigits (in base 10) and showed that the largest such number is $F_{14}=377$. Recently, diophantine equations involving Padovan numbers and repdigits have also been studied. In Ref. [12], the authors found all repdigits that can be written as a sum of two Padovan numbers. This result was later extended to repdigits that are a sum of three Padovan numbers by the second author in Ref. [8]. In Ref. [9], in the direction similar to the one in Ref. [1], the second author considered all Padovan numbers that can be written as a concatenation of two distinct repdigits and showed that the largest such number is $P_{21}=200$. More specifically, it was shown that if $P_{n}$ is a solution of the Diophantine equation $P_{n}=\underbrace{\overline{d_{1} \cdots d_{1}} \underbrace{d_{2} \cdots d_{2}}_{m \text { times }}}_{\ell \text { times }}$, times, then

$$
P_{n} \in\{12,16,21,28,37,49,65,86,114,200\} .
$$

Other related interesting results in this research direction include: the result of Bednařík and Trojovská [3], the result of Boussayoud, et al. [4], the result of Bravo and Luca [5], the result of the second author [7], the result of Erduvan and Keskin [11], the result of Rayaguru and Panda [16], the results of Trojovský [17,18], and the result of Qu and Zeng [15]. A natural continuation of the result in Ref. [9] would be a characterization of palindromic Padovan numbers. As a first step in this direction, we (for the time being) consider the (more restrictive) Diophantine equation

$$
\begin{aligned}
P_{n}= & \underbrace{}_{\begin{array}{l}
\ell \text { times } \\
\text { where }
\end{array}} \underbrace{d_{1} \cdots d_{1}, d_{2}}_{m \text { times }} \in\{0,1,2, \ldots, 9\}, \quad d_{1}>0 .
\end{aligned}
$$

Our result is the following.

Theorem 1 The only Padovan numbers which are palindromic concatenations of two distinct repdigits are

$$
P_{n} \in\{151,616\}
$$

\section{Preliminary results}

In this section we collect some facts about Padovan numbers and other preliminary lemmas that are crucial to our main argument. This preamble to the main result is similar to the one in Ref. [9] and is included here for the sake of completeness. 


\subsection{Some properties of the Padovan numbers}

Recall that the characteristic equation of the Padovan sequence is given by $\phi(x):=x^{3}-$ $x-1=0$, with roots $\alpha, \beta$, and $\gamma=\bar{\beta}$ given by:

$$
\alpha=\frac{r_{1}+r_{2}}{6} \text { and } \beta=\frac{-\left(r_{1}+r_{2}\right)+i \sqrt{3}\left(r_{1}-r_{2}\right)}{12},
$$

where

$$
r_{1}=\sqrt[3]{108+12 \sqrt{69}} \text { and } r_{2}=\sqrt[3]{108-12 \sqrt{69}}
$$

For all $n \geq 0$, Binet's formula for the Padovan sequence tells us that the $n$th Padovan number is given by

$$
P_{n}=a \alpha^{n}+b \beta^{n}+c \gamma^{n}
$$

where

$$
a=\frac{\alpha+1}{(\alpha-\beta)(\alpha-\gamma)}, \quad b=\frac{\beta+1}{(\beta-\alpha)(\beta-\gamma)}, \quad \text { and } \quad c=\frac{\gamma+1}{(\gamma-\alpha)(\gamma-\beta)}=\bar{b} .
$$

The minimal polynomial of $a$ over $\mathbb{Z}$ is given by

$$
23 x^{3}-5 x-1,
$$

and its zeros are $a, b, c$ as given above. One can check that $|a|,|b|,|c|<1$. Numerically, we have the following estimates for the quantities $\{\alpha, \beta, \gamma, a, b, c\}$ :

$$
\begin{gathered}
1.32<\alpha<1.33 \\
0.86<|\beta|=|\gamma|=\alpha^{-\frac{1}{2}}<0.87 \\
0.54<a<0.55 \\
0.28<|b|=|c|<0.29
\end{gathered}
$$

It follows that the contribution to the right hand side of Eq. (2) due to the complex conjugate roots $\beta$ and $\gamma$ is small. More specifically, let

$$
e(n):=P_{n}-a \alpha^{n}=b \beta^{n}+c \gamma^{n} \text {. Then, }|e(n)|<\frac{1}{\alpha^{n / 2}} \text { for all } n \geq 1 .
$$

The last inequality in (4) follows from the fact that $|\beta|=|\gamma|=\alpha^{-\frac{1}{2}}$ and $|b|=|c|<0.29$ (by (3)). That is, for any $n \geq 1$,

$$
|e(n)|=\left|b \beta^{n}+c \gamma^{n}\right| \leq|b||\beta|^{n}+|c||\gamma|^{n}=|b| \alpha^{-\frac{n}{2}}+|c| \alpha^{-\frac{n}{2}}<2 \cdot 0.29 \cdot \alpha^{-\frac{n}{2}}<\frac{1}{\alpha^{n / 2}} .
$$

Furthermore, the following estimate also holds:

Lemma 1 Let $n \geq 1$ be a positive integer. Then

$$
\alpha^{n-3} \leq P_{n} \leq \alpha^{n-1} .
$$

Lemma 1 follows from a simple inductive argument.

Let $\mathbb{K}:=\mathbb{Q}(\alpha, \beta)$ be the splitting field of the polynomial $\phi$ over $\mathbb{Q}$. Then $[\mathbb{K}: \mathbb{Q}]=6$ and $[\mathbb{Q}(\alpha): \mathbb{Q}]=3$. We note that the Galois group of $\mathbb{K} / \mathbb{Q}$ is given by

$$
\mathcal{G}:=\operatorname{Gal}(\mathbb{K} / \mathbb{Q}) \cong\{(1),(\alpha \beta),(\alpha \gamma),(\beta \gamma),(\alpha \beta \gamma)\} \cong S_{3}
$$


Therefore, we identify the automorphisms of $\mathcal{G}$ with the permutation group of the zeroes of $\phi$. We shall find particular use for the permutation $(\alpha \beta)$, corresponding to the automorphism $\sigma: \alpha \mapsto \beta, \beta \mapsto \alpha, \gamma \mapsto \gamma$.

\subsection{Linear forms in logarithms}

Like many proofs of similar results, the crucial steps in our argument involve obtaining certain bounds on linear forms in (nonzero) logarithms. The upper bounds usually follow easily from a manipulation of the associated Binet's formula for the sequence in question. For the lower bounds, we need the celebrated Baker's theorem on linear forms in logarithms. Before stating the result, we need the definition of the (logarithmic) Weil height of an algebraic number. Let $\eta$ be an algebraic number of degree $d$ with minimal polynomial

$$
P(x)=a_{0} \prod_{j=1}^{d}\left(x-\alpha_{j}\right),
$$

where the leading coefficient $a_{0}$ is positive and the $\alpha_{j}$ 's are the conjugates of $\alpha$. The logarithmic height of $\eta$ is given by

$$
h(\eta):=\frac{1}{d}\left(\log a_{0}+\sum_{j=1}^{d} \log \left(\max \left\{\left|\alpha_{j}\right|, 1\right\}\right)\right) .
$$

Note that, if $\eta=\frac{p}{q} \in \mathbb{Q}$ is a reduced rational number with $q>0$, then the above definition reduces to $h(\eta)=\log \max \{|p|, q\}$. We list some well known properties of the height function below, which we shall subsequently use without reference:

$$
\begin{aligned}
h\left(\eta_{1} \pm \eta_{2}\right) & \leq h\left(\eta_{1}\right)+h\left(\eta_{2}\right)+\log 2, \\
h\left(\eta_{1} \eta_{2}^{ \pm 1}\right) & \leq h\left(\eta_{1}\right)+h\left(\eta_{2}\right), \\
h\left(\eta^{s}\right) & =|s| h(\eta), \quad(s \in \mathbb{Z}) .
\end{aligned}
$$

We quote the version of Baker's theorem proved by Bugeaud, Mignotte, and Siksek ([6], Theorem 9.4).

Theorem 2 Let $\eta_{1}, \ldots, \eta_{t}$ be positive real algebraic numbers in a real algebraic number field $\mathbb{K} \subset \mathbb{R}$ of degree $D$. Let $b_{1}, \ldots, b_{t}$ be nonzero integers such that

$$
\Gamma:=\eta_{1}^{b_{1}} \ldots \eta_{t}^{b_{t}}-1 \neq 0 .
$$

Then

$$
\log |\Gamma|>-1.4 \cdot 30^{t+3} \cdot t^{4.5} \cdot D^{2}(1+\log D)(1+\log B) A_{1} \ldots A_{t},
$$

where

$$
B \geq \max \left\{\left|b_{1}\right|, \ldots,\left|b_{t}\right|\right\}
$$

and

$$
A_{j} \geq \max \left\{D h\left(\eta_{j}\right),\left|\log \eta_{j}\right|, 0.16\right\}, \text { for all } j=1, \ldots, t \text {. }
$$




\subsection{Baker-Davenport reduction}

The bounds on the variables obtained via Baker's theorem are usually too large for any computational purposes. In order to get further refinements, we use the Baker-Davenport reduction procedure. The variant we apply here is the one due to Dujella and Pethö ([10], Lemma 5a). For a real number $r$, we denote by $\|r\|$ the quantity $\min \{|r-n|: n \in \mathbb{Z}\}$, which is the distance from $r$ to the nearest integer.

Lemma 2 Let $\kappa \neq 0$, and $A, B, \mu$ be real numbers with $A>0$ and $B>1$. Let $M>1$ be $a$ positive integer and suppose that $\frac{p}{q}$ is a convergent of the continued fraction expansion of $\kappa$ with $q>6 M$. Let

$$
\varepsilon:=\|\mu q\|-M\|\kappa q\| .
$$

If $\varepsilon>0$, then there is no solution of the inequality

$$
0<|m \kappa-n+\mu|<A B^{-k}
$$

in positive integers $m, n, k$ with

$$
\frac{\log (A q / \varepsilon)}{\log B} \leq k \text { and } m \leq M .
$$

We will also need the following lemma by Gúzman Sánchez and Luca ([13], Lemma 7):

Lemma 3 Let $r \geq 1$ and $H>0$ be such that $H>\left(4 r^{2}\right)^{r}$ and $H>L /(\log L)^{r}$. Then

$$
L<2^{r} H(\log H)^{r} .
$$

\section{Proof of the main result}

\subsection{The low range}

With the help of a simple computer program in Mathematica, we checked all the solutions to the Diophantine equation (1) in the ranges $d_{1} \neq d_{2} \in\{0,1,2, \ldots, 9\}, d_{1}>0$ and $1 \leq \ell, m \leq n \leq 1000$. We found only the solutions stated in Theorem 1 . Here onwards, we assume that $n>1000$.

\subsection{The initial bound on $n$}

We note that (1) can be rewritten as

$$
\begin{aligned}
& P_{n}=\overline{\underbrace{d_{1} \cdots d_{1}}_{\ell \text { times }} \underbrace{d_{2} \cdots d_{2}}_{m \text { times }} \underbrace{d_{1} \cdots d_{1}}_{\ell \text { times }}} \\
& =\underbrace{d_{1} \cdots d_{1}}_{\ell \text { times }} \cdot 10^{\ell+m}+\underbrace{d_{2} \cdots d_{2}}_{m \text { times }} \cdot 10^{\ell}+\underbrace{d_{1} \cdots d_{1}}_{\ell \text { times }} \\
& =\frac{1}{9}\left(d_{1} \cdot 10^{2 \ell+m}-\left(d_{1}-d_{2}\right) \cdot 10^{\ell+m}+\left(d_{1}-d_{2}\right) \cdot 10^{\ell}-d_{1}\right) .
\end{aligned}
$$

The next lemma relates the sizes of $n$ and $2 \ell+m$. 
Lemma 4 All solutions of (5) satisfy

$$
(2 \ell+m) \log 10-3<n \log \alpha<(2 \ell+m) \log 10+1 .
$$

Proof Recall that $\alpha^{n-3} \leq P_{n} \leq \alpha^{n-1}$. We note that

$$
\alpha^{n-3} \leq P_{n}<10^{2 \ell+m} .
$$

Taking the logarithm on both sides, we get

$$
n \log \alpha<(2 \ell+m) \log 10+3 \log \alpha .
$$

Hence, $n \log \alpha<(2 \ell+m) \log 10+1$. The lower bound follows via the same technique, upon noting that $10^{2 \ell+m-1}<P_{n} \leq \alpha^{n-1}$.

We proceed to examine (5) in three different steps as follows.

Step 1. From Eqs. (2) and (5), we have that

$$
9\left(a \alpha^{n}+b \beta^{n}+c \gamma^{n}\right)=d_{1} \cdot 10^{2 \ell+m}-\left(d_{1}-d_{2}\right) \cdot 10^{\ell+m}+\left(d_{1}-d_{2}\right) \cdot 10^{\ell}-d_{1} .
$$

Hence,

$$
9 a \alpha^{n}-d_{1} \cdot 10^{2 \ell+m}=-9 e(n)-\left(d_{1}-d_{2}\right) \cdot 10^{\ell+m}+\left(d_{1}-d_{2}\right) \cdot 10^{\ell}-d_{1} .
$$

We thus have that

$$
\begin{aligned}
\left|9 a \alpha^{n}-d_{1} \cdot 10^{2 \ell+m}\right| & =\left|-9 e(n)-\left(d_{1}-d_{2}\right) \cdot 10^{\ell+m}+\left(d_{1}-d_{2}\right) \cdot 10^{\ell}-d_{1}\right| \\
& \leq 9 \alpha^{-n / 2}+27 \cdot 10^{\ell+m} \\
& <28 \cdot 10^{\ell+m}
\end{aligned}
$$

where we used the fact that $n>1000$. Dividing both sides by $d_{1} \cdot 10^{2 \ell+m}$, we get

$$
\left|\left(\frac{9 a}{d_{1}}\right) \cdot \alpha^{n} \cdot 10^{-2 \ell-m}-1\right|<\frac{28 \cdot 10^{m+\ell}}{d_{1} \cdot 10^{2 \ell+m}} \leq \frac{28}{10^{\ell}} .
$$

We put

$$
\Gamma_{1}:=\left(\frac{9 a}{d_{1}}\right) \cdot \alpha^{n} \cdot 10^{-2 \ell-m}-1 .
$$

We shall compare this upper bound on $\left|\Gamma_{1}\right|$ with the lower bound we deduce from Theorem 2. Note that $\Gamma_{1} \neq 0$, since this would imply that $a \alpha^{n}=\frac{d_{1} \cdot 10^{2 \ell+m}}{9}$. If this is the case, then applying the automorphism $\sigma$ on both sides of the preceeding equation and taking absolute values, we have that

$$
\left|\frac{d_{1} \cdot 10^{2 \ell+m}}{9}\right|=\left|\sigma\left(a \alpha^{n}\right)\right|=\left|b \beta^{n}\right|<1,
$$

which is false. We thus have that $\Gamma_{1} \neq 0$.

With a view towards applying Theorem 2, we define the following parameters:

$$
\eta_{1}:=\frac{9 a}{d_{1}}, \eta_{2}:=\alpha, \eta_{3}:=10, b_{1}:=1, b_{2}:=n, b_{3}:=-2 \ell-m, t:=3 .
$$

Since $10^{2 \ell+m-1}<P_{n} \leq \alpha^{n-1}$, we have that $2 \ell+m<n$. Thus we take $B=n$. We note that $\mathbb{K}=\mathbb{Q}\left(\eta_{1}, \eta_{2}, \eta_{3}\right)=\mathbb{Q}(\alpha)$, since $a=\alpha(\alpha+1) /(2 \alpha+3)$. Hence $D=[\mathbb{K}: \mathbb{Q}]=3$. 
We note that

$$
h\left(\eta_{1}\right)=h\left(\frac{9 a}{d_{1}}\right) \leq 2 h(9)+h(a) \leq 2 \log 9+\frac{1}{3} \log 23<5.44 .
$$

We also have that $h\left(\eta_{2}\right)=h(\alpha)=\frac{1}{3} \log \alpha$ and $h\left(\eta_{3}\right)=\log 10$. Hence, we let

$$
A_{1}:=16.32, A_{2}:=\log \alpha, A_{3}:=3 \log 10 .
$$

Thus, we deduce via Theorem 2 that

$$
\begin{aligned}
\log |\Gamma| & >-1.4 \cdot 30^{6} 3^{4.5} 3^{2}(1+\log 3)(1+\log n)(16.32)(\log \alpha)(3 \log 10) \\
& >-1.45 \cdot 10^{30}(1+\log n) .
\end{aligned}
$$

Comparing the last inequality obtained above with (6), we get

$$
\ell \log 10-\log 28<1.45 \cdot 10^{30}(1+\log n),
$$

and therefore,

$$
\ell \log 10<1.46 \cdot 10^{30}(1+\log n) .
$$

Step 2. We rewrite Eq. (5) as

$$
9 a \alpha^{n}-d_{1} \cdot 10^{2 \ell+m}+\left(d_{1}-d_{2}\right) \cdot 10^{m+\ell}=-9 e(n)+\left(d_{1}-d_{2}\right) \cdot 10^{\ell}-d_{1} .
$$

That is,

$$
9 a \alpha^{n}-\left(d_{1} \cdot 10^{\ell}-\left(d_{1}-d_{2}\right)\right) \cdot 10^{m+\ell}=-9 e(n)+\left(d_{1}-d_{2}\right) \cdot 10^{\ell}-d_{1} .
$$

Hence,

$$
\begin{aligned}
\left|9 a \alpha^{n}-\left(d_{1} \cdot 10^{\ell}-\left(d_{1}-d_{2}\right)\right) \cdot 10^{m+\ell}\right| & =\left|-9 e(n)+\left(d_{1}-d_{2}\right) \cdot 10^{\ell}-d_{1}\right| \\
& \leq \frac{9}{\alpha^{n / 2}}+18 \cdot 10^{\ell}<19 \cdot 10^{\ell}
\end{aligned}
$$

Dividing throughout by $\left(d_{1} \cdot 10^{\ell}-\left(d_{1}-d_{2}\right)\right) \cdot 10^{m+\ell}$, we have that

$$
\left|\left(\frac{9 a}{d_{1} \cdot 10^{\ell}-\left(d_{1}-d_{2}\right)}\right) \cdot \alpha^{n} \cdot 10^{-\ell-m}-1\right|<\frac{19 \cdot 10^{\ell}}{\left(d_{1} \cdot 10^{\ell}-\left(d_{1}-d_{2}\right)\right) \cdot 10^{m+\ell}}<\frac{19}{10^{m}} \text {. }
$$

We put

$$
\Gamma_{2}:=\left(\frac{9 a}{d_{1} \cdot 10^{\ell}-\left(d_{1}-d_{2}\right)}\right) \cdot \alpha^{n} \cdot 10^{-\ell-m}-1 .
$$

As before, we have that $\Gamma_{2} \neq 0$ because this would imply that

$$
a \alpha^{n}=10^{m+\ell}\left(\frac{d_{1} \cdot 10^{\ell}-\left(d_{1}-d_{2}\right)}{9}\right),
$$

which in turn implies that

$$
\left|10^{m+\ell}\left(\frac{d_{1} \cdot 10^{\ell}-\left(d_{1}-d_{2}\right)}{9}\right)\right|=\left|\sigma\left(a \alpha^{n}\right)\right|=\left|b \beta^{n}\right|<1,
$$


which is false. In preparation towards applying Theorem 2, we define the following parameters:

$\eta_{1}:=\frac{9 a}{d_{1} \cdot 10^{\ell}-\left(d_{1}-d_{2}\right)}, \eta_{2}:=\alpha, \eta_{3}:=10, b_{1}:=1, b_{2}:=n, b_{3}:=-\ell-m, t:=3$.

In order to determine what $A_{1}$ will be, we need to find the find the maximum of the quantities $h\left(\eta_{1}\right)$ and $\left|\log \eta_{1}\right|$.

We note that

$$
\begin{aligned}
h\left(\eta_{1}\right) & =h\left(\frac{9 a}{d_{1} \cdot 10^{\ell}-\left(d_{1}-d_{2}\right)}\right) \\
& \leq h(9)+h(a)+\ell h(10)+h\left(d_{1}\right)+h\left(d_{1}-d_{2}\right)+\log 2 \\
& \leq 3 \log 9+h(a)+\ell \log 10 \\
& <3 \log 9+\frac{1}{3} \log 23+1.46 \cdot 10^{30}(1+\log n) \\
& <1.48 \cdot 10^{30}(1+\log n),
\end{aligned}
$$

where in the second last inequality above, we used (7). On the other hand, we also have that

$$
\begin{aligned}
\left|\log \eta_{1}\right| & =\left|\log \left(\frac{9 a}{d_{1} \cdot 10^{\ell}-\left(d_{1}-d_{2}\right)}\right)\right| \\
& \leq \log a+\log 9+\left|\log \left(d_{1} \cdot 10^{\ell}-\left(d_{1}-d_{2}\right)\right)\right| \\
& \leq \log a+\log 9+\log \left(d_{1} \cdot 10^{\ell}\right)+\left|\log \left(1-\frac{d_{1}-d_{2}}{d_{1} \cdot 10^{\ell}}\right)\right| \\
& \leq \ell \log 10+\log d_{1}+\log 9+\log (0.55)+\left|\frac{\left|d_{1}-d_{2}\right|}{d_{1} \cdot 10^{\ell}}+\frac{1}{2}\left(\frac{\left|d_{1}-d_{2}\right|}{d_{1} \cdot 10^{\ell}}\right)^{2}+\cdots\right| \\
& \leq \ell \log 10+3 \log 9+\frac{1}{10^{\ell}}+\frac{1}{2 \cdot 10^{2 \ell}}+\cdots \\
& \leq 1.46 \cdot 10^{30}(1+\log n)+3 \log 9+\frac{1}{10^{\ell}-1} \\
& <1.48 \cdot 10^{30}(1+\log n),
\end{aligned}
$$

where in the second last inequality, we used Eq. (7). We note that $D h\left(\eta_{1}\right)>\left|\log \eta_{1}\right|$. We thus let $A_{1}:=4.44 \cdot 10^{30}(1+\log n)$. We take $A_{2}:=\log \alpha$ and $A_{3}:=3 \log 10$, as defined in Step 1. Similarly, we take $B:=n$.

Theorem 2 then tells us that

$$
\begin{aligned}
\log \left|\Gamma_{2}\right| & >-1.4 \cdot 30^{6} \cdot 3^{4.5} \cdot 3^{2} \cdot(1+\log 3)(1+\log n)(\log \alpha)(3 \log 10) A_{1} \\
& >-2 \cdot 10^{13}(1+\log n) A_{1}>-8.88 \cdot 10^{43}(1+\log n)^{2} .
\end{aligned}
$$

Comparing the last inequality with (8), we have that

$$
\begin{aligned}
m \log 10 & <8.88 \cdot 10^{43}(1+\log n)^{2}+\log 19 \\
& <9 \cdot 10^{43}(1+\log n)^{2} .
\end{aligned}
$$

Step 3. We rewrite Eq. (5) as

$$
9 a \alpha^{n}-d_{1} \cdot 10^{2 \ell+m}+\left(d_{1}-d_{2}\right) \cdot 10^{m+\ell}-\left(d_{1}-d_{2}\right) \cdot 10^{\ell}=-9 e(n)-d_{1} .
$$


Therefore,

$$
\begin{aligned}
\left|9 a \alpha^{n}-\left(d_{1} \cdot 10^{\ell+m}+\left(d_{1}-d_{2}\right) \cdot 10^{m}-\left(d_{1}-d_{2}\right)\right) \cdot 10^{\ell}\right| & =\left|-9 e(n)-d_{1}\right| \\
& \leq \frac{9}{\alpha^{n / 2}}+9<10 .
\end{aligned}
$$

Consequently,

$$
\left|\left(\frac{1}{9 a}\right)\left(d_{1} \cdot 10^{\ell+m}+\left(d_{1}-d_{2}\right) \cdot 10^{m}-\left(d_{1}-d_{2}\right)\right) \cdot \alpha^{-n} \cdot 10^{\ell}-1\right|<\frac{10}{9 a \alpha^{n}}<\frac{3}{\alpha^{n}} .
$$

Let

$$
\Gamma_{3}:=\left[\left(\frac{1}{9 a}\right)\left(d_{1} \cdot 10^{\ell+m}+\left(d_{1}-d_{2}\right) \cdot 10^{m}-\left(d_{1}-d_{2}\right)\right)\right] \cdot \alpha^{-n} \cdot 10^{\ell}-1 .
$$

As before, we have that $\Gamma_{3} \neq 0$ since we would have that

$$
a \alpha^{n}=\frac{1}{9}\left(d_{1} \cdot 10^{\ell+m}+\left(d_{1}-d_{2}\right) \cdot 10^{m}-\left(d_{1}-d_{2}\right)\right) \cdot 10^{\ell} .
$$

Applying the automorphism $\sigma$ from the Galois group $\mathcal{G}$ on both sides of the above equation and then taking absolute values, we have that

$$
\left|\frac{1}{9}\left(d_{1} \cdot 10^{\ell+m}+\left(d_{1}-d_{2}\right) \cdot 10^{m}-\left(d_{1}-d_{2}\right)\right) \cdot 10^{\ell}\right|=\left|\sigma\left(a \alpha^{n}\right)\right|=\left|b \beta^{n}\right|<1,
$$

which is false. We would now like to apply Theorem 2 to $\Gamma_{3}$. To this end, we let:

$$
\begin{gathered}
\eta_{1}:=\left[\left(\frac{1}{9 a}\right)\left(d_{1} \cdot 10^{\ell+m}+\left(d_{1}-d_{2}\right) \cdot 10^{m}-\left(d_{1}-d_{2}\right)\right)\right], \eta_{2}:=\alpha, \eta_{3}:=10, \\
b_{1}:=1, b_{2}:=-n, b_{3}:=\ell, t:=3 .
\end{gathered}
$$

As in the previous cases, we can take $B:=n$ and $D:=3$. We note that

$$
\begin{aligned}
h\left(\eta_{1}\right) \leq & h(9)+h(a)+h\left(d_{1}\right)+(\ell+m) h(10)+h\left(d_{1}-d_{2}\right) \\
& +m h(10)+h\left(d_{1}-d_{2}\right)+3 \log 2 \\
\leq & 5 \log 9+\frac{\log 23}{3}+(\ell+m) \log 10+m \log 10 \\
\leq & 6 \log 9+(\ell+m) \log 10+m \log 10 .
\end{aligned}
$$

Using Eqs. (7) and (9), we have that

$$
\begin{aligned}
(\ell+m) \log 10 & <1.46 \cdot 10^{30}(1+\log n)+9 \cdot 10^{43}(1+\log n)^{2} \\
& <10 \cdot 10^{43}(1+\log n)^{2} .
\end{aligned}
$$

Thus, we deduce that

$$
h\left(\eta_{1}\right)<20 \cdot 10^{43}(1+\log n)^{2} .
$$


We now find an upper bound for $\left|\log \eta_{1}\right|$. We have that

$$
\begin{aligned}
& \left|\log \eta_{1}\right|=\left|\log \left(\left(\frac{1}{9 a}\right)\left(d_{1} \cdot 10^{\ell+m}+\left(d_{1}-d_{2}\right) \cdot 10^{m}-\left(d_{1}-d_{2}\right)\right)\right)\right| \\
& \leq \log 9+\log a+\left|\log \left(d_{1} \cdot 10^{\ell+m}+\left(d_{1}-d_{2}\right) \cdot 10^{m}-\left(d_{1}-d_{2}\right)\right)\right| \\
& \leq 2 \log 9+\log \left(d_{1} \cdot 10^{\ell+m}\right)+\left|\log \left(1-\frac{\left(d_{1}-d_{2}\right)\left(10^{m}-1\right)}{d_{1} \cdot 10^{\ell+m}}\right)\right| \\
& \leq 3 \log 9+(\ell+m) \log 10+\left|\log \left(1-\frac{\left(d_{1}-d_{2}\right)\left(10^{m}-1\right)}{d_{1} \cdot 10^{\ell+m}}\right)\right| \\
& \leq 3 \log 9+(\ell+m) \log 10 \\
& +\left|\frac{\left|\left(d_{1}-d_{2}\right)\left(10^{m}-1\right)\right|}{d_{1} \cdot 10^{\ell+m}}+\frac{1}{2}\left(\frac{\left|\left(d_{1}-d_{2}\right)\left(10^{m}-1\right)\right|}{d_{1} \cdot 10^{\ell+m}}\right)^{2}+\cdots\right| \\
& \leq 3 \log 9+(\ell+m) \log 10+\frac{1}{10^{\ell}}+\frac{1}{2 \cdot 10^{2 \ell}}+\cdots \\
& <3 \log 9+(\ell+m) \log 10+\frac{1}{10^{\ell}-1} \\
& <1.1 \cdot 10^{44}(1+\log n)^{2},
\end{aligned}
$$

where in the last inequality above, we used the bound from (11). We note that $D \cdot h\left(\eta_{1}\right)>$ $\left|\log \eta_{1}\right|$. We thus let $A_{1}:=6 \cdot 10^{44}(1+\log n)^{2}, A_{2}:=\log \alpha$ and $3 \log 10$. Theorem 2 then implies that

$$
\log \left|\Gamma_{3}\right|>-2 \cdot 10^{13}(1+\log n) A_{1}>-1.2 \cdot 10^{58}(1+\log n)^{3} .
$$

Comparing the last inequality with (10), we deduce that

$$
n \log \alpha<1.2 \cdot 10^{58}(1+\log n)^{3}+\log 3 .
$$

It follows that

$$
n<5 \cdot 10^{58}(\log n)^{3} .
$$

With the notation of Lemma 3, we let $r:=3, L:=n$, and $H:=5 \cdot 10^{58}$ and notice that this data meets the conditions of the lemma. Applying the lemma, we have that

$$
n<2^{3} \cdot 5 \cdot 10^{58}\left(\log \left(5 \cdot 10^{58}\right)\right)^{3} .
$$

After a simplification, we obtain the (rather loose) bound

$$
n<1.04 \cdot 10^{66} \text {. }
$$

Lemma 4 then implies that

$$
2 \ell+m<1.4 \cdot 10^{65} .
$$

The following lemma summarizes what we have proved thus far:

Lemma 5 All solutions to the Diophantine equation (1) satisfy

$$
2 \ell+m<1.4 \cdot 10^{65} \text { and } n<1.04 \cdot 10^{66} .
$$




\subsection{The reduction procedure}

The bounds obtained in Lemma 5 are too large to be useful computationally. Thus, we need to reduce them. To do so, we apply Lemma 2 as follows. First, we return to the inequality (6) and put

$$
z_{1}:=(2 \ell+m) \log 10-n \log \alpha+\log \left(\frac{d_{1}}{9 a}\right) .
$$

The inequality (6) can be rewritten as

$$
\left|\Gamma_{1}\right|=\left|e^{-z_{1}}-1\right|<\frac{28}{10^{\ell}} .
$$

If we assume that $\ell \geq 2$, then the right-hand side of the above inequality is at most 28/100 < $1 / 2$. The inequality $\left|e^{z}-1\right|<x$ for real values of $x$ and $z$ implies that $z<2 x$. Thus,

$$
\left|z_{1}\right|<\frac{56}{10^{\ell}} \text {. }
$$

This implies that

$$
\left|(2 \ell+m) \log 10-n \log \alpha-\log \left(\frac{9 a}{d_{1}}\right)\right|<\frac{56}{10^{\ell}} .
$$

Dividing through the above inequality by $\log \alpha$ gives

$$
\left|(2 \ell+m) \frac{\log 10}{\log \alpha}-n+\left(\frac{\log \left(d_{1} / 9 a\right)}{\log \alpha}\right)\right|<\frac{56}{10^{\ell} \log \alpha} .
$$

So, we apply Lemma 2 with the quantities:

$$
\kappa:=\frac{\log 10}{\log \alpha}, \quad \mu\left(d_{1}\right):=\frac{\log \left(d_{1} / 9 a\right)}{\log \alpha}, \quad 1 \leq d_{1} \leq 9, \quad A:=\frac{56}{\log \alpha}, \quad B:=10 .
$$

Let $\kappa=\left[a_{0} ; a_{1}, a_{2}, \ldots\right]=[8 ; 5,3,3,1,5,1,8,4,6,1,4,1,1,1,9,1,4,4,9,1,5, \ldots]$ be the continued fraction expansion of $\kappa$. We set $M:=10^{66}$ which is the upper bound on $2 \ell+m$. With the help of Mathematica, we find that the convergent

$$
\frac{p}{q}=\frac{p_{141}}{q_{141}}=\frac{92894276795199235673676174009251522651329656614011503595729035741839}{11344567100398997770258435239827426964781308977543724537727298754290},
$$

is such that $q=q_{141}>6 M$. Furthermore, it gives $\varepsilon>0.0716554$, and thus,

$$
\ell \leq \frac{\log ((56 / \log \alpha) q / \varepsilon)}{\log 10}<70 .
$$

Therefore, we have that $\ell \leq 70$. The case $\ell<2$ also holds because $\ell<2<70$.

Next, for fixed $d_{1} \neq d_{2} \in\{0,1,2, \ldots, 9\}, d_{1}>0$ and $1 \leq \ell \leq 70$, we return to the inequality (8) and put

$$
z_{2}:=(\ell+m) \log 10-n \log \alpha+\log \left(\frac{d_{1} \cdot 10^{\ell}-\left(d_{1}-d_{2}\right)}{9 a}\right) .
$$

From the inequality (8), we have that

$$
\left|\Gamma_{2}\right|=\left|e^{-z_{2}}-1\right|<\frac{19}{10^{m}} .
$$


Assume that $m \geq 2$, then the right-hand side of the above inequality is at most $19 / 100<1 / 2$. Thus, we have that

$$
\left|z_{2}\right|<\frac{38}{10^{m}}
$$

which implies that

$$
\left|(\ell+m) \log 10-n \log \alpha+\log \left(\frac{d_{1} \cdot 10^{\ell}-\left(d_{1}-d_{2}\right)}{9 a}\right)\right|<\frac{38}{10^{m}} .
$$

Dividing through by $\log \alpha$ gives

$$
\left|(\ell+m) \frac{\log 10}{\log \alpha}-n+\frac{\log \left(\left(d_{1} \cdot 10^{\ell}-\left(d_{1}-d_{2}\right)\right) / 9 a\right)}{\log \alpha}\right|<\frac{38}{10^{m} \log \alpha} .
$$

Thus, we apply Lemma 2 with the quantities:

$$
\mu\left(d_{1}, d_{2}\right):=\frac{\log \left(\left(d_{1} \cdot 10^{\ell}-\left(d_{1}-d_{2}\right)\right) / 9 a\right)}{\log \alpha}, \quad A:=\frac{38}{\log \alpha}, \quad B:=10 .
$$

We take the same $\kappa$ and its convergent $p / q=p_{141} / q_{141}$ as before. Since $\ell+m<2 \ell+m$, we set $M:=10^{66}$ as the upper bound on $\ell+m$. With the help of a simple computer program in Mathematica, we get that $\varepsilon>0.0000918806$, and therefore,

$$
m \leq \frac{\log ((38 / \log \alpha) q / \varepsilon)}{\log 10}<73 .
$$

Thus, we have that $m \leq 73$. The case $m<2$ holds as well since $m<2<73$.

Lastly, for fixed $d_{1} \neq d_{2} \in\{0,1,2, \ldots, 9\}, d_{1}>0,1 \leq \ell \leq 69$ and $1 \leq m \leq 73$, we return to the inequality (10) and put

$$
z_{3}:=\ell \log 10-n \log \alpha+\log \left(\frac{d_{1} \cdot 10^{\ell+m}+\left(d_{1}-d_{2}\right) \cdot 10^{m}-\left(d_{1}-d_{2}\right)}{9 a}\right) .
$$

From the inequality (10), we have that

$$
\left|\Gamma_{3}\right|=\left|e^{z_{3}}-1\right|<\frac{3}{\alpha^{n}} .
$$

Since $n>1000$, the right-hand side of the above inequality is less than $1 / 2$. Thus, the above inequality implies that

$$
\left|z_{3}\right|<\frac{6}{\alpha^{n}}
$$

which leads to

$$
\left|\ell \log 10-n \log \alpha+\log \left(\frac{d_{1} \cdot 10^{\ell+m}+\left(d_{1}-d_{2}\right) \cdot 10^{m}-\left(d_{1}-d_{2}\right)}{9 a}\right)\right|<\frac{6}{\alpha^{n}} .
$$

Dividing through by $\log \alpha$ gives,

$$
\left|\ell \frac{\log 10}{\log \alpha}-n+\frac{\log \left(\left(d_{1} \cdot 10^{\ell+m}+\left(d_{1}-d_{2}\right) \cdot 10^{m}-\left(d_{1}-d_{2}\right)\right) / 9 a\right)}{\log \alpha}\right|<\frac{6}{\alpha^{n} \log \alpha} .
$$


Again, we apply Lemma 2 with the quantities:

$$
\mu\left(d_{1}, d_{2}\right):=\frac{\log \left(\left(d_{1} \cdot 10^{\ell+m}+\left(d_{1}-d_{2}\right) \cdot 10^{m}-\left(d_{1}-d_{2}\right)\right) / 9 a\right)}{\log \alpha}, \quad A:=\frac{6}{\log \alpha}, \quad B:=\alpha .
$$

We take the same $\kappa$ and its convergent $p / q=p_{141} / q_{141}$ as before. Since $\ell<2 \ell+m$, we choose $M:=10^{66}$ as the upper bound for $\ell$. With the help of a simple computer program in Mathematica, we get that $\varepsilon>0.00000594012$, and thus,

$$
n \leq \frac{\log ((6 / \log \alpha) q / \varepsilon)}{\log \alpha}<602 .
$$

Thus, we have shown that $n \leq 602$, contradicting our assumption that $n>1000$. Therefore, Theorem 1 holds.

Acknowledgements The authors of this paper thank the anonymous referees and the editor for the careful reading of the manuscript and the useful comments and suggestions that greatly improved the quality of presentation of the current paper.

Funding Open access funding provided by Austrian Science Fund (FWF). M.D. was supported by FWF projects: F05510-N26 - Part of the special research program (SFB), "Quasi-Monte Carlo Methods: Theory and Applications" and W1230 - "Doctoral Program Discrete Mathematics".

\section{Declarations}

Conflict of interest The authors declare that they have no conflict of interest.

Open Access This article is licensed under a Creative Commons Attribution 4.0 International License, which permits use, sharing, adaptation, distribution and reproduction in any medium or format, as long as you give appropriate credit to the original author(s) and the source, provide a link to the Creative Commons licence, and indicate if changes were made. The images or other third party material in this article are included in the article's Creative Commons licence, unless indicated otherwise in a credit line to the material. If material is not included in the article's Creative Commons licence and your intended use is not permitted by statutory regulation or exceeds the permitted use, you will need to obtain permission directly from the copyright holder. To view a copy of this licence, visit http://creativecommons.org/licenses/by/4.0/.

\section{References}

1. Alahmadi, A., Altassan, A., Luca, F., Shoaib, H.: Fibonacci numbers which are concatenations of two repdigits. Quaest. Math. 44(2), 281-290 (2021). https://doi.org/10.2989/16073606.2019.1686439

2. Banks, W.D., Luca, F.: Concatenations with binary reccurent sequences. J. Integer Seq. 8(5), Art. 05.1.3 (2005)

3. Bednařík, D., Trojovská, E.: Repdigits as product of Fibonacci and Tribonacci numbers. Mathematics 8(10), 1720 (2020). https://doi.org/10.3390/math8101720

4. Boussayoud, A., Boughaba, S., Kerada, M., Araci, S., Acikgoz, M.: Generating functions of binary products of $k$-Fibonacci and orthogonal polynomials. Rev. R. Acad. Cienc. Exactas Fís. Nat. Ser. A Mat. RACSAM 113(3), 2575-2586 (2019). https://doi.org/10.1007/s13398-019-00641-4

5. Bravo, J.J., Luca, F.: On a conjecture about repdigits in $k$-generalized Fibonacci sequences. Publ. Math. Debreceen 82(3-4), 623-639 (2013)

6. Bugeaud, Y., Mignotte, M., Siksek, S.: Classical and modular approaches to exponential Diophantine equations I. Fibonacci and Lucas perfect powers. Ann. Math. 163(2), 969-1018 (2006)

7. Ddamulira, M.: Tribonacci numbers that are concatenations of two repdigits. Rev. R. Acad. Cienc. Exactas Fís. Nat. Ser. A Mat. RACSAM 114(4), Paper No. 203, 10 pp (2020) https://doi.org/10.1007/s13398020-00933-0 
8. Ddamulira, M.: Repdigits as sums of three Padovan numbers. Bol. Soc. Mat. Mex. 26(2), 247-261 (2020). https://doi.org/10.1007/s40590-019-00269-9

9. Ddamulira, M.: Padovan numbers that are concatenations of two distinct repdigits. Math. Slovaca 71(2), 275-284 (2021). https://doi.org/10.1515/ms-2017-0467

10. Dujella, A., Pethő, A.: A generalization of a theorem of Baker and Davenport. Q. J. Math. Oxford Ser. 2 49(195), 291-306 (1998)

11. Erduvan, F., Keskin, R.: Lucas numbers which are concatenations of three repdigits. Results Math. 76(1), 13 (2021). https://doi.org/10.1007/s00025-020-01314-0

12. Garcíá Lomelí, A.C., Hernández Hernández, S.: Repdigits as sums of two Padovan numbers. J. Integer Seq. 22(2), Art. 19.2.3 (2019)

13. Gúzman Sánchez, S., Luca, F.: Linear combinations of factorials and $s$-units in a binary recurrence sequence. Ann. Math. Qué. 38(2), 169-188 (2014). https://doi.org/10.1007/s40316-014-0025-z

14. Luca, F.: Fibonacci and Lucas numbers with only one distinct digit. Portugal. Math. 57(2), 243-254 (2000)

15. Qu, Y., Zeng, J.: Lucas numbers which are concatenations of two repdigits. Mathematics 8(8), 1360 (2020). https://doi.org/10.3390/math8081360

16. Rayaguru, S.G., Panda, G.K.: Balancing numbers which are concatenations of two repdigits. Bol. Soc. Mat. Mex. 26(3), 911-919 (2020). https://doi.org/10.1007/s40590-020-00293-0

17. Trojovský, P.: Fibonacci numbers with a prescribed block of digits. Mathematics 8(4), 639 (2020). https:// doi.org/10.3390/math8040639

18. Trojovský, P.: On repdigits as sums of Fibonacci and Tribonacci numbers. Symmetry 12(11), 1774 (2020). https://doi.org/10.3390/sym12111774

Publisher's Note Springer Nature remains neutral with regard to jurisdictional claims in published maps and institutional affiliations. 